\title{
ON THE ORDER OF THE PARTIAL SUMS OF A FOURIER SERIES*
}

W. C. RANDELS

We propose to show here that the known estimate $S_{n}=o(n)$ cannot be improved. To do this it is sufficient to show that there exists a sequence of functions $f_{n}(x)$ for which there is a constant $A$ such that,

$$
S_{n}\left(f_{n}, 0\right)>A n,
$$

and

$$
\begin{gathered}
S_{\nu}\left(f_{n}, 0\right) \rightarrow 0 \text { as } \nu \rightarrow \infty, \\
\int_{-\pi}^{\pi}\left|f_{n}(x) d x\right|<1 .
\end{gathered}
$$

For, if (1), (2), and (3) are satisfied, then for every sequence of positive numbers $d_{n}$, with $d_{n} \rightarrow 0$ as $n \rightarrow \infty, \lim \sup n d_{n}=\infty$, we can choose a sequence of integers $n_{i}$ such that

$$
\left|\sum_{j=1}^{i-1} d_{n_{j}} S_{n_{i}}\left(f_{n_{j}}, 0\right)\right|<\frac{A}{3} n_{i} d_{n_{i}}
$$

and

$$
d_{n_{i+1}}<\frac{A}{3 \pi} d_{n_{i}}
$$

We notice that

(6) $S_{n_{j}}\left(f_{n_{i}}, 0\right)=\frac{\pi}{2} \int_{-\pi}^{\pi} f_{n_{i}}(x) D_{n_{j}}(x) d x<\pi n_{j} \int_{-\pi}^{\pi}\left|f_{n_{i}}(x)\right| d x<\pi n_{j}$,

and this implies that the constant $A$ in (1) is less than $\pi$. Then, if $f(x)$ is defined by

$$
f(x)=\sum_{i=1}^{\infty} d_{n_{i}} f_{n_{i}}(x),
$$

$f(x) \subset L$, since from (3) and (5)

$$
\int_{-\pi}^{\pi}|f(x)| d x \leqq \sum_{i=1}^{\infty} d_{n_{i}} \int_{-\pi}^{\pi}\left|f_{n_{i}}(x)\right| d x<\frac{d_{1} A}{\pi} \sum_{i=1}^{\infty} 3^{-i}=\frac{d_{1} A}{2 \pi} .
$$

* Presented to the Society, April 10, 1937. 
We have also

$$
\begin{aligned}
\left|S_{n_{i}}(f, 0)\right| & =\left|\sum_{j=1}^{\infty} d_{n_{j}} S_{n_{i}}\left(f_{n_{j}}, 0\right)\right| \\
& =\left|\sum_{j=1}^{i-1} d_{n_{j}} S_{n_{i}}\left(f_{n_{j}}, 0\right)+d_{n_{i}} S_{n_{j}}\left(f_{n_{i}}, 0\right)+\sum_{j=i+1}^{\infty} d_{n_{j}} S_{n_{i}}\left(f_{n_{i}}, 0\right)\right| \\
& >A n_{i} d_{n_{i}}-\frac{1}{3} A n_{i} d_{n_{i}}-\pi n_{i} \sum_{j=i+1}^{\infty} d_{n_{j}} \\
& >\frac{1}{3} A n_{i} d_{n_{i}} .
\end{aligned}
$$

We shall now prove the existence of a sequence $f_{n}(x)$ with the properties (1), (2), and (3). We define

$$
f_{n}(x)=\left\{\begin{array}{l}
n / 2, \pi / 4\left(n+\frac{1}{2}\right) \leqq|x| \leqq \pi / 2\left(n+\frac{1}{2}\right), \\
0, \text { elsewhere. }
\end{array}\right.
$$

Then

$$
\int_{-\pi}^{\pi}\left|f_{n}(x)\right| d x=\frac{n \pi}{8\left(n+\frac{1}{2}\right)}<1,
$$

and, since $f_{n}(x)$ vanishes in the neighborhood of the origin,

$$
S_{\nu}\left(f_{n}, 0\right) \rightarrow 0 \quad \text { as } \quad \nu \rightarrow \infty \text {. }
$$

Finally,

$$
\begin{aligned}
S_{n}\left(f_{n}, 0\right) & =\pi \int_{0}^{\pi} f_{n}(x) \frac{\sin \left(n+\frac{1}{2}\right) x}{\sin x / 2} d x \\
& =\frac{n \pi}{2} \int_{\pi(n+1 / 2) / 4}^{\pi / 2(n+1 / 2)} \frac{\sin \left(n+\frac{1}{2}\right) x}{\sin x / 2} d x \\
& >\frac{\pi n}{2} \cdot \frac{\pi}{4\left(n+\frac{1}{2}\right)} \cdot \frac{2\left(n+\frac{1}{2}\right)}{\pi}>\pi n / 4,
\end{aligned}
$$

and our theorem is proved.

We shall merely mention that similar results could be easily obtained for the Cesàro sums of a Fourier series. For the sequence of functions we have constructed satisfies (1), (2), and (3) when $S_{n}(f, x)$ is replaced by $S_{n}{ }^{\alpha}(f, x)$ where $S_{n}{ }^{\alpha}(f, x)$ is the $C_{\alpha}$ transform of the Fourier series of $f(x)$.

We shall now point out a consequence of this result.* We know, of

* The author is indebted to Professor Szász for pointing this out. 
course, that the Fourier series of a function of bounded variation converges everywhere; we are now able to state that nothing can be said about the order of convergence even if we restrict ourselves to absolutely continuous functions. For let us suppose that there is some sequence $c_{n},\left(c_{n} \rightarrow 0\right)$, such that for all absolutely continuous $F(x)$

$$
S_{n}(F, x)-F(x)=O\left(C_{n}\right) .
$$

Let $f(x)$ be the function defined above, and define

$$
f_{a}(x)=f(a+x)-f(a-x) .
$$

Then

$$
f_{a}(x) \sim \sum_{n=1}^{\infty} b_{n} \sin n x
$$

and

$$
S_{n_{i}}\left(f_{a}, a\right)>\frac{A}{3} n_{i} d_{n_{i}}-o(1) \neq o\left(n_{i} d_{n_{i}}\right) .
$$

If $F(x)$ is defined by

$$
F(x)=\int_{0}^{x} f(x) d x
$$

we have

$$
F(x) \sim \frac{A_{0}}{2}+\sum_{n=1}^{\infty} A_{n} \cos n x,
$$

where $A_{n}=b_{n} / n, n \geqq 1$. Then, if ( 7$)$ is true,

$$
\begin{aligned}
S_{n}\left(f_{a}, x\right)= & \sum_{\nu=1}^{n} \nu A_{\nu} \sin \nu x \\
= & {\left[-S_{0}(F, x)+F(x)\right] \sin \dot{x}+\left[S_{n}(F, x)-F(x)\right] n \sin n x } \\
& +\sum_{\nu=1}^{n-1}\left[S_{\nu}(F, x)-F(x)\right][\nu \sin \nu x-(\nu+1) \sin (\nu+1) x] \\
= & O\left(n C_{n}\right) .
\end{aligned}
$$

But by (8) the $d_{n}$ can be chosen so that this is impossible, and therefore (7) must be false.

NORTHWESTERN UNIVERSITY 